\title{
L'Homme
}

Revue française d'anthropologie

$218 \mid 2016$

Varia

\section{De deux modalités de comparaison en anthropologie sociale}

Going Full Frontal, or the Elision of Lateral Comparison in Anthropology

\section{Matei Candea}

Traducteur : Franck Lemonde

\section{OpenEdition}

Journals

Édition électronique

URL : http://journals.openedition.org/lhomme/28968

DOI : 10.4000//homme.28968

ISSN : 1953-8103

Éditeur

Éditions de l'EHESS

\section{Édition imprimée}

Date de publication : 20 mai 2016

Pagination : 183-218

ISSN : 0439-4216

\section{Référence électronique}

Matei Candea, "De deux modalités de comparaison en anthropologie sociale », L'Homme [En ligne],

218 | 2016, mis en ligne le 19 mai 2018, consulté le 10 décembre 2020. URL : http://

journals.openedition.org//homme/28968; DOl : https://doi.org/10.4000//homme.28968

(c) École des hautes études en sciences sociales 


\title{
De deux modalités de comparaison en anthropologie sociale
}

\author{
Matei Candea \\ "Ce qu'il nous faut vraiment éviter, ce ne sont pas les erreurs, \\ mais les erreurs décisives que nous ne pouvons pas corriger. \\ Même les erreurs décisives sont tolérables tant qu'elles sont faciles à déceler"
}

(Wimsatt 2007: 24)

L

LA DÉMARCHE classique en anthropologie, qui consiste à lire ses données de terrain à travers le prisme de l'opposition entre un groupe humain déterminé et l' "Occident", est depuis longtemps la cible d'une critique vigoureuse (par exemple, Said 2003 [1978] ; Fabian 2006 [1983] ; Carrier 1992; Pina Cabral 2006). On ne saurait mieux présenter ces critiques qu'en citant l'analyse sans concession d'un anthropologue qui continue néanmoins à utiliser ces dualismes :

"Au terme de cette introduction, je me dois d'insérer une remarque sur mon propre usage des concepts de l' "Occidental" et du "moderne". Ces concepts n’ont pas cessé d'être source de tracas pour les anthropologues, et je ne fais pas exception. À chaque fois que je me surprends en train de les utiliser, je me mords les lèvres de frustration, et j'aurais préféré les éviter. Les objections à ces concepts sont bien connues : à savoir que, dans la plupart des études anthropologiques, ils jouent un rôle souvent implicite de repoussoir permettant de faire ressortir un "point de vue indigène"; qu' une bonne partie de l'outillage philosophique servant à la critique de la pensée dite occidentale ou moderne provient directement de la tradition occidentale elle-même (des figures comme le jeune Karl Marx, Martin Heidegger et Maurice Merleau-Ponty se trouvent alors enrôlées pour montrer combien les conceptions des Indiens d'Amérique du Nord, des montagnards de Nouvelle-Guinée ou des Aborigènes d'Australie different de celles des "Euro-Américains"); qu'une fois que nous connaissons bien les personnes - même les habitants des pays appelés occidentaux -, aucune d'entre elles ne s'avère être Occidentale de souche, ni même être particulièrement moderne dans sa vision du monde, et que la tradition de pensée occidentale, vue de près, est aussi richement variée, multivoque, historiquement changeante et conflictuelle qu'une autre " (Ingold $2000: 6-7)$.

Ce texte est la traduction révisée d'une communication présentée en septembre 2015 au séminaire "The History of Cross-Cultural Comparatism: Modern Doubts and New Beginnings ", au Centre for Research in the Arts, Social Sciences and Humanities, de Cambridge. 
Et pourtant, en dépit de ces attaques renouvelées et de ces griefs aujourd'hui bien connus, non seulement l'usage d'" essentialisations binaires dialectiquement produites" (Carrier 1992) subsiste toujours en anthropologie, mais il est, de plus, apparemment en plein essor ${ }^{1}$. Dans cet article, je montrerai que les faiblesses tout autant que la persistance paradoxale de cette forme d'argumentation anthropologique s'expliquent mieux si on la considère comme faisant partie d'un couple indissociable d'heuristiques comparatives. Cette " comparaison frontale ", dans laquelle une entité ethnographique étrangère est mise en contraste avec un milieu prétendument familier, est intimement mêlée et nécessairement associée aux «comparaisons latérales $»^{2}$, où un certain nombre de «cas " sont juxtaposés. La première partie de cet article développera ce contraste. Dans la deuxième, j'examinerai comment la comparaison frontale a trouvé un second souffle après son discrédit épistémologique des années 1980. La troisième partie se concentrera sur la dynamique par laquelle, tandis que la comparaison frontale a monopolisé l'attention et les préoccupations épistémiques des anthropologues ces dernières décennies, les discussions méthodologiques et épistémologiques autour de la comparaison latérale ont été abandonnées, étant jugées comme appartenant à un positivisme désuet - à quelques exceptions près (Pedersen \& Nielsen 2013; Strathern 2004 [1991]). Le regain d'intérêt pour la comparaison frontale est en partie un effet de cette inégalité de visibilité - la comparaison latérale se maintient, certes, mais en fonctionnant le plus souvent sans surveillance épistémique, pour soutenir et contrebalancer son pendant frontal bien plus visible. La quatrième et dernière partie analysera cette persistance, cette vitalité et cette diversité de la comparaison latérale à partir de

1. Pour ne prendre que deux exemples récents assez typiques, Rane Willerslev soutient que : «C'est un présupposé communément partagé en Occident que les attributs de la personnalité, avec tout ce que cela inclut en termes de langage, d'intentionnalité, de raisonnement et de conscience morale, appartiennent exclusivement aux êtres humains. On conçoit les animaux comme des êtres entièrement naturels, et leur comportement est d'ordinaire expliqué comme automatique et instinctif. Néanmoins, chez les Yukaghirs, un présupposé différent prévaut. Dans leur monde, les personnes peuvent adopter des formes diverses, dont les êtres humains ne sont qu'une partie" (2004: 629). Tandis que Viveiros de Castro prétend que : «Le chamanisme est une forme d'agir qui présuppose un type de savoir, un certain idéal de connaissance. Cet idéal, à bien des égards, est l'exact opposé de l'épistémologie populaire objectiviste de notre tradition. Selon celle-ci, c'est la catégorie de l'objet qui fournit le telos : connaitre, c'est objectiver [...]. La forme de l'autre, c'est la chose. Le chamanisme amérindien est guidé par l'idéal opposé. Connaître, c'est personnifier, adopter le point de vue de ce qui doit être connu. La connaissance chamanique vise quelque chose qui est quelqu'un - un autre sujet. La forme de l'autre, c'est la personne» (2004a : 468). Même les contrastes radicaux de Louis Dumont, longtemps tenus pour la quintessence de l'essentialisme «nous/eux» (cf. Pina Cabral 2006), sont en train d'être redécouverts comme prescients, subtils et pertinents (Iteanu \& Moya 2015).

2. Pour une conception de la comparaison latérale légèrement différente de celle proposée ici, cf. Christopher Gad (2012) et Christopher Gad \& Casper Bruun Jensen (2016). 
la lecture attentive d'un exemple précis : l'article de Carlos Fausto sur le cannibalisme en Amazonie (2007). En conclusion, je reviendrai sur l'interdépendance de ces deux heuristiques, pour défendre l'idée qu'il est temps de remettre la comparaison latérale en pleine lumière.

En qualifiant les comparaisons frontale et latérale d'heuristiques, je n'entends pas ce terme au sens faible et vague que certains auteurs lui donnent pour servir leurs propres argumentations. Dire que certaines affirmations ou démarches sont "purement heuristiques " peut en effet être une façon commode d'éluder les défauts et limites de certains arguments plutôt que de s'y confronter. Pour ma part, j'emploie le terme heuristique au sens technique élaboré par le philosophe des sciences William Wimsatt. L'heuristique, selon Wimsatt, est un ensemble de procédés conceptuels ${ }^{3}$ qui ont six caractéristiques (2007: 346) :

1) ils n'offrent pas de garanties;

2) ils sont rentables;

3) ils sont systématiquement biaisés ;

4) ils transforment un problème en un autre problème non équivalent mais intuitivement lié ;

5) ils sont reliés à un objectif;

6) ils sont issus d'autres heuristiques.

La référence habituelle à l'heuristique comme moyen d'esquiver les problèmes ne comprend que les points 1 et 2 . Mais la notion commence vraiment à s'imposer comme outil pour penser la théorie et la pratique avec le point 3 : les heuristiques sont systématiquement biaisées. C'està-dire qu'elles ne sont pas simplement défaillantes, elles le sont de manière régulière et prédictible. Wimsatt décrit cette forme caractéristique d'erreur comme l'" empreinte" de l'heuristique (Ibid.: 80). Le fait d'avoir une empreinte constante est l'une des dimensions principales de la valeur de l'heuristique. Cela permet de discerner à quelles fins une heuristique est utile, et à quelles fins elle sera systématiquement fallacieuse (cf. le point 5). Cela permet aussi de combiner différentes heuristiques qui, ensemble, contribueront à l'élaboration d'une image "solide" ${ }^{4}$ (Ibid.: 44).

3. Plus que conceptuels. Wimsatt intègre la notion d'heuristique dans des réflexions plus larges d'ingénierie et d'épistémologie évolutionniste. Les procédés mécaniques ou computationnels sont également de l'heuristique. Au sens élargi, toute "adaptation" évolutionniste est une heuristique. Naturellement, il n'est pas nécessaire d'accepter les autres dimensions de l'épistémologie évolutionniste pour utiliser la notion d'heuristique dans le sens de Wimsatt.

4. Il est intéressant de constater que Wimsatt fait de la "solidité " (robustness), non seulement un critère épistémologique, mais également un critère ontologique. De fait, pour Wimsatt, les entités sont plus « réelles » à mesure qu’elles sont plus solides. 
À l'inverse, cela permet d'éviter de s'appuyer sur un ensemble d'heuristiques dont les signes distinctifs se recouperaient dans une large mesure - qui auraient systématiquement le même mode de défaillance ${ }^{5}-$, tendant ainsi à produire une impression factice de solidité.

La tentative de Wimsatt de "réagencer» la philosophie des sciences à partir de notions comme l'heuristique offre aux anthropologues un certain nombre de ressources remarquablement fécondes et jusqu’à présent inexploitées. Bien que je n’aie pas la place nécessaire pour développer ici la plupart de ces suggestions, celles-ci ont une portée significative à la fois sur le plan épistémologique et sur le plan ontologique. Le simple fait de prendre au sérieux la notion d'heuristique telle qu'on l'a définie plus haut pourrait contribuer à modifier la manière dont les débats sont menés au sein de notre discipline. Traiter nos propres concepts, techniques et présupposés, et ceux des autres, comme autant d'heuristiques perfectibles a deux effets positifs. Le premier est de garder à l'esprit qu'ils ne sont "que» des heuristiques - évitant ainsi les débats quelque peu fastidieux suscités par des propos grandiloquents sur la seule manière, ou la meilleure, ou la plus morale de faire de l'anthropologie. Une heuristique échoue quand elle oublie qu'elle n'est qu'une heuristique. Le second effet positif est de nous rappeler que les concepts, les approches (etc.) sont au moins heuristiques. Autrement dit, ils sont susceptibles de se montrer utiles malgré et même grâce à leurs points faibles. Diagnostiquer ces points faibles devient, non pas un "travail de sape " des autres approches ou paradigmes (Navaro-Yashin 2009), une "critique tout-ou-rien ", mais plutôt la reconnaissance d'avantages provisoires et de réussites fragiles (Anderson 2001: 132; Pina-Cabral 2009).

La distinction entre comparaisons latérale et frontale est elle-même, bien sûr, une distinction heuristique - par là, comme je pense l'avoir fait comprendre plus haut, je ne veux pas dire que je cherche à m'en tirer aussi facilement. Bien au contraire, j'espère susciter des questions que vous garderez à l'esprit pendant votre lecture : qu'obtient-on par cette distinction ? Et à quel prix ? Si, finalement, ma distinction heuristique entre comparaisons frontale et latérale n'était pas jugée utile, du moins aurons-nous réalisé ensemble le genre d'exercice (penser par l'heuristique) que cet article essaye de susciter et d'illustrer.

5. Ainsi, Wimsatt expose d'une façon assez éclairante comment un grand nombre d'heuristiques réductionnistes, qui semblent toutes très différentes, échouent toutes systématiquement à détecter les effets contextuels. 


\section{Comparaisons frontale et latérale}

La meilleure manière de présenter le contraste entre la comparaison frontale et la comparaison latérale est de revenir sur une vieille controverse. Dans son article fondateur qui attaquait le programme de RadcliffeBrown visant à faire de l'anthropologie une science naturelle de la société, Evans-Pritchard décrivait la pratique des anthropologues comme un ensemble d'opérations séquentielles et logiquement distinctes. Dans la première " phase ", l'ethnologue :

" [...] va s'établir au milieu d'un peuple primitif et s'initie à leur mode de vie. Il apprend à parler leur langue, à penser en fonction de leurs concepts et à réagir suivant leur échelle de valeurs. Puis il revoit son expérience en fonction des concepts et du système de valeurs qui sont ceux de sa propre culture et des connaissances particulières à la discipline qu'il pratique. En d'autres termes, il fait la traduction d'une culture en une autre» (1969 [1951] : 80).

Dans la deuxième phase, l'anthropologue «essaye d'aller au-delà du stade littéraire et impressionniste afin de découvrir l'ordre structural de la société » qu'il étudie (Ibid.: 81, voir aussi 1950 : 122). Enfin, dans la troisième phase, l'anthropologue " une fois qu'il a isolé les grandes lignes de la structure dans une société, [...] va les comparer avec celles d'autres sociétés" (Ibid.). Comme il l'explique dans une discussion plus approfondie, ce qu'Evans-Pritchard a en tête ici n'est pas cette pratique de l'armchair anthropology que l'on appelait alors communément la "méthode comparative", mais plutôt une procédure lente et progressive, adaptée à une discipline dont les praticiens étaient d'abord et avant tout des enquêteurs de terrain. Un anthropologue conduit une étude pour arriver à certaines conclusions (par exemple sur le rôle des cultes religieux dans la vie sociale) :

« S'il les formule clairement et en termes tels qu'ils puissent se subdiviser en un certain nombre d'autres sujets de recherche, il est dorénavant possible à un autre anthropologue, ou au même, de faire auprès d'une seconde population des observations qui démontreront si ces conclusions sont généralisables ou non. Il s’apercevra que certaines sont vérifiées, d'autres non et que les autres nécessitent des modifications. En partant du point atteint par la première étude, la seconde sera vraisemblablement plus poussée et apportera de nouvelles confirmations aux conclusions de la première [...]. On fait alors une troisième étude, puis une quatrième, puis une cinquième. On peut continuer indéfiniment " (Ibid. : 113-114).

Cette présentation d'Evans-Pritchard peut servir de référence pour mesurer les changements intervenus dans notre façon de concevoir la discipline. Il semblerait en effet que presque tous les aspects de sa description 
de la pratique de l'anthropologue aient été démontés dans les années qui ont suivi. La conviction avec laquelle Evans-Pritchard suggère que la première phase rend la société "culturellement intelligible" aux yeux d'un étranger comme elle le serait aux yeux d'un de ses membres; la conviction encore plus absolue selon laquelle la seconde phase rend la société "sociologiquement intelligible»; l’image de sociétés comme étant des unités stables nettement délimitées pour des analyses successives; l'idée de catégories comparatives (par exemple, "cultes religieux") qui ne demandent qu'une formulation précise pour servir de support à une enquête cumulative; le test d'hypothèses; l'intérêt ou la possibilité de l'abstraction structurelle; la possibilité ou l'aspiration à une approche holistique des sociétés; l'idée même que le but du travail anthropologique est d'obtenir des similitudes et de formuler des généralisations provisoires. Tels sont les éléments principaux du tableau d'Evans-Pritchard qui ont été remis en question, ensemble ou séparément, et avec plus ou moins de succès, par différentes critiques internes à la discipline.

Quels qu'aient pu être les éléments abandonnés depuis, le propos d'Evans-Pritchard élabore un contraste essentiel qui demeure intact à ce jour, à savoir la complémentarité entre deux différentes "démarches » ou directions de la comparaison anthropologique. Définissons la comparaison, en termes très généraux, comme la démarche qui consiste à rapprocher deux entités différentes afin de produire un effet (épistémique, ontologique, politique, etc.) par un examen de leurs différences et similitudes. À ce niveau d'abstraction, on peut dire que la présentation d'Evans-Pritchard commence par une comparaison frontale entre un " autre » ethnographique et le "milieu " d'où vient l'ethnographe. Elle finit par une comparaison latérale dans laquelle différents cas anthropologiques sont confrontés les uns aux autres.

L'idée selon laquelle ces deux démarches représentent des "phases" consécutives dans une seule analyse - ou même, comme le dit EvansPritchard, des «niveaux d'abstraction" consécutifs (1950:122) - ne résiste pas à l'examen. De fait, l'exposé d'Evans-Pritchard brise lui-même cette périodisation tranchée. Après tout, quand l'ethnographe, dans la première phase, revit son expérience de terrain à la lumière du corpus de connaissances propres à sa discipline, n'est-il pas déjà en train de s'inspirer du type d'exercice décrit dans la troisième "phase » ? Néanmoins, le fait que les démarches frontales et latérales soient entrelacées à chaque étape de construction du savoir anthropologique, et que ce processus ne puisse être nettement décomposé en étapes successives, ne réduit pas la valeur heuristique de la distinction d'Evans-Pritchard. C'est ce que je voudrais maintenant développer. 
À première vue, la différence entre comparaisons frontale et latérale pourrait sembler facile à résumer : la première est un ensemble de comparaisons entre "eux" et "nous", alors que la seconde est un ensemble de comparaisons entre "eux" et "eux" et "eux", etc. La comparaison frontale ne serait dès lors qu'un cas particulier de comparaison latérale, dans laquelle une des entités impliquées se trouve être la "société d'origine " de l'anthropologue lui-même. Mais cela masque une différence plus profonde entre les deux formes de comparaison, que nous pouvons clairement reconstituer à partir de l'analyse d'Evans-Pritchard : alors que la comparaison latérale mobilise des entités qui sont formellement du même ordre, quoique différentes dans leur contenu, la comparaison frontale mobilise des entités qui sont constitutivement différentes dans leur forme - en fait, constitutivement asymétriques. Le «nous» dans la comparaison frontale n'est pas le même type d'entité que le «eux».

Du côté "eux» de la comparaison frontale, il y a un objet ethnographique : une expérience vécue, personnelle, que l'ethnologue va s'efforcer de décrire et d'analyser pour des lecteurs qui en ignorent tout. Du côté "nous", se trouvent «les concepts et [le] système de valeurs qui sont ceux de sa propre culture et des connaissances particulières à la discipline [que l'ethnographe] pratique» (Evans-Pritchard 1969 [1951] : 80). Par opposition à l'objet ethnographique, qui est une partie d'un vaste territoire inexploré « là-bas au loin », j'appellerai cette position du "nous » l'« hinterland». L'hinterland est la terre d'origine de l'ethnographe, ce sont les rivages conceptuels d'où il a embarqué initialement, avec, dans ses bagages, l'outillage intellectuel qu'il partage (en partie) avec ses lecteurs, et à partir duquel il émettra ses hypothèses et tirera ses conclusions. Ainsi, l'apparente symétrie de deux "cultures» ou "ontologies» se faisant face cache à la vue de tous le fait que la position «nous", ne requiert jamais autant d'élaboration que la position "eux». Par définition, la position du «nous» est supposée être déjà partagée et peut ainsi n'être esquissée que succinctement. Les anthropologues peuvent parfois insister, pour leur public, sur des aspects particuliers de leur hinterland " partagé » (les postures occidentales vis-à-vis de la connaissance objective, des conceptions de la personne humaine ou des animaux, par exemple), ils peuvent même puiser dans certaines références classiques ou choisir des citations significatives pour étayer ces généralisations, mais ils n'ont guère besoin d'apporter beaucoup plus de précisions à leurs descriptions ${ }^{6}$.

6. «En ce qui concerne les principes généraux de notre cosmologie partagée, ce n’est pas tant un défaut d'information qu'il faut éventuellement combler, ainsi que je me suis attaché à le faire dans le cas de l'animisme ou du totémisme, mais bien plutôt une connaissance surabondante qu'il convient d'épurer pour en retrouver les lignes directrices" (Descola $2005: 244$ ). 
L'hinterland est par ailleurs systématiquement partiel et, dans une large mesure, secondaire pour l'ethnographie. Tel qu'il est invoqué dans une ethnographie, l'hinterland inclut seulement les aspects de «notre " milieu qui sont pertinents par contraste avec l'expérience ethnographique. L'hinterland est constitué de ces parties de nous-mêmes que l'ethnographie altérise en nous contraignant à les expliciter. C'est l'ombre projetée par l'ethnographie sur notre propre milieu, qu'elle révèle précisément comme milieu. Considérés ensemble, ce point et le précédent conduisent à une situation subtilement paradoxale. L'hinterland est, par définition, ce que l'anthropologue partage avec ses lecteurs, et, pourtant, au moment même où il est rendu explicite, il ne correspond plus tout à fait à la position de l'anthropologue lui-même. L'hinterland est à la fois cette terre partagée entre l'anthropologue et ses lecteurs et la marque de ce qui les sépare, puisque l'anthropologue, par son ethnographie, rend visible l'hinterland en tant que simple hinterland.

Enfin, de même que ce public est double (Evans-Pritchard concevait un lectorat composé d'anthropologues et d'autres Occidentaux, ou simplement d'anthropologues eux-mêmes occidentaux), de même l'hinterland est un mélange de présupposés à la fois culturels et propres à la discipline - point important sur lequel je reviendrai plus loin.

Ainsi la comparaison frontale s'effectue à partir d'entités constitutivement asymétriques : un objet ethnographique qui requiert l'élucidation et un hinterland qui agit simultanément comme cadre et comme contrepoint à cette élucidation. En revanche, la comparaison latérale s'effectue à partir d'entités de même nature, qui ne sont pas des "sociétés " en tant que telles - Evans-Pritchard n'est pas un empiriste naïf -, mais plutôt des "études" de sociétés : des entités hybrides résultant de la description d'une société ou d'un aspect d'une société par un ethnographe particulier, largement imprégné des problèmes et des catégories propres à sa discipline. Les éléments de construction de la comparaison latérale, les unités à partir desquelles elle est mise en œuvre, sont les résultats d'une opération antérieure de comparaison frontale (phase 1) et d'analyse structurale (phase 2). Leur utilité à des fins comparatives vient de leur diversité substantielle en termes de contenu et de leur ressemblance en termes de forme. Les études, pour être comparativement pertinentes, doivent résulter de pratiques similaires, avoir été entreprises soit par le même ethnographe ${ }^{7}$, soit au moins par les membres reconnus d'une

7. Il peut sembler étonnant pour le lecteur contemporain, habitué au récit classique selon lequel l'anthropologie n'est devenue réflexive qu'autour de 1980, qu'Evans-Pritchard ait pu analyser avec tant de précision le degré de dépendance de toute étude ethnographique envers la personnalité individuelle et l'idiosyncrasie de son auteur. 
communauté épistémique partageant les mêmes méthodes, catégories ou objets de recherche. Les entités impliquées dans la comparaison latérale - les études - peuvent être différentes en qualité ou en valeur bien sûr, mais elles sont toutes accessibles (en tant qu'études) par l'ensemble des lecteurs.

Il est possible que la distinction latéral/frontal rappelle celle établie par certains chercheurs entre les traditions « interprétiviste " (ou " humaniste ») et "positiviste" (ou scientiste) en anthropologie (Holý, ed. 1987; Handler 2009; Lazar 2012). Mais ces deux distinctions présentent plusieurs différences fondamentales. Tandis que l'opposition positiviste/ interprétiviste cherche à restituer un « changement de paradigme » (Holý), les comparaisons frontale et latérale sont des heuristiques qui sont bien plus anciennes et bien plus rudimentaires. Les comparaisons frontale et latérale ne sont pas des approches mutuellement exclusives en anthropologie, elles sont fréquemment sinon toujours imbriquées dans le tissu de chaque description anthropologique. Cette distinction est également, dans une large mesure, indépendante des autres distinctions habituellement établies entre le positivisme et l'interprétivisme (en matière d'objectif, de méthode, de conception des unités à comparer, de présupposés épistémologiques, etc.)

Par exemple, la présentation d'Evans-Pritchard met en évidence le fait que ces monuments de la comparaison positiviste latérale que sont les Systèmes politiques africains (Fortes \& Evans-Pritchard 1964 [1940]) et Systèmes familiaux et matrimoniaux en Afrique (Radcliffe-Brown \& Forde 1953 [1950]) reposaient sur une comparaison frontale faite par chaque ethnographe pour construire son propre cas. Chaque volume en appelle à une remise en question frontale de "nos " conceptions de l'État ou de la famille, comme le montrent leurs préfaces respectives. Les grands classiques structuralistes ou néostructuralistes de la comparaison latérale (Lévi-Strauss 1962; Descola 2005) s'appuient, de leur côté, sur une logique latérale très différente, comme on l'a souvent relevé (Salmon 2013). Mais, là aussi, en quelque sorte, nous voyons la comparaison frontale jouer un rôle constitutif à la fois en amont et en aval du travail. Quant aux «interprétivistes ", Holý relève lui-même que l'un des moyens - éminemment frontal - par lesquels ils cherchaient à élucider la spécificité culturelle était de comparer différentes pratiques au sein d'un même contexte culturel pour en montrer les points communs (Holý 1987 : 12) - comparaison latérale s'il en est.

Il est vrai, et je reviendrai sur ce point, que le clivage positiviste/ interprétiviste peut être en partie caractérisé comme un clivage dans l'attention prêtée respectivement à ces deux modalités de comparaison. 
Comme Holý l'a remarqué, les anthropologues qui travaillent dans le cadre de traditions considérées comme herméneutiques se sont souvent frottés à la question de la rencontre frontale entre l'observateur et l'observé, tandis que ceux qui sont habituellement qualifiés de positivistes tendent à mettre l'accent sur le travail latéral à partir d'autres cas. Mais même cette vision est incomplète. D'une part, comme je le dirai plus bas, l'accent mis sur la frontalité s'est reporté sur de nouvelles traditions anthropologiques, comme celle du tournant ontologique. D'autre part, même à l'intérieur du paysage traditionnellement concerné par la distinction positiviste/interprétiviste, il existe des exceptions importantes et fréquentes. Les écrits de Malinowski sur la science, la religion et la magie chez les Trobriands et en Occident, notamment (Malinowski 1925), sont un exemple classique de comparaison frontale avec de fortes tendances positivistes, tandis que la plus grande partie du travail de Max Weber, pour prendre à nouveau un exemple des plus classiques, est à la fois herméneutique et profondément engagée dans la comparaison latérale.

En somme, on peut dire provisoirement et de façon très générale que, considérées comme procédés heuristiques, les comparaisons frontale et latérale jouent des rôles clairement distincts, en anthropologie comme ailleurs. La comparaison frontale est le procédé heuristique qui accomplit cette double démarche bien connue de l'élucidation anthropologique : rendre le familier étrange et l'étrange familier. Cette démarche est bien sûr bien plus ancienne que l'anthropologie elle-même, comme cela a souvent été remarqué (Trouillot 1991) - c'est une forme d'argument ayant une longue histoire, qui remonte au moins à Montaigne, voire, de façon plus discontinue, à Hérodote (Hartog 1980).

On conçoit fréquemment la comparaison latérale, qui est elle aussi une heuristique éminemment ancienne, comme aspirant à "généraliser » à partir de cas particuliers, afin d'établir des lois locales ou plus générales - c'est à l'évidence la conception d'auteurs comme Radcliffe-Brown (Nadel 1951; Radcliffe-Brown 1951). Dans cette approche, la comparaison latérale consiste à établir des similitudes à partir de cas divers. Mais la description d'Evans-Pritchard nous permet de prendre du recul et de comprendre qu'il ne s'agit que d'un aspect de la comparaison latérale. Cette dernière, comme le montre bien Evans-Pritchard, peut tout autant accentuer les différences - par de nouvelles questions, variations, possi-bilités et autres sujets de recherche. C'est pourquoi, tandis que le processus d'identification des similitudes à partir de différents cas apparaît généra-lement comme une sorte de point d'aboutissement satisfaisant ou suffisant de l'analyse, dans la conception plus ouverte d'EvansPritchard, la comparaison latérale " peut continuer indéfiniment» (1969 
[1951] : 114). Ici, le but n'est pas d'arriver à une conclusion générale, mais, plus poussé » (Ibid.).

\section{Du frontal, rien que du frontal?}

"[The] device of the straw man obviated any need for a comparative view "

(Strathern 1981: 666).

Evans-Pritchard décrit lui-même la démarche frontale comme une "traduction " culturelle et c'est la démarche latérale qui est, pour lui, assimilable à la méthode comparative. Pour mon propos, l'analogie avec la traduction linguistique (voir Ingold 1993 ; Pina Cabral 1992 ; Asad 1986) soulève des questions qui nous lancent sur une fausse piste. Toutefois, cette perspective est un bon outil pour diagnostiquer la persistance de la distinction entre comparaisons frontale et latérale en anthropologie. Nous trouvons ainsi une suite ininterrompue de discussions sur la "traduction culturelle", depuis les étudiants et les collaborateurs d'Evans-Pritchard (Beattie 1972 [1964]; Lienhardt 1953), en passant par les critiques de Talal Asad (1986), jusqu'à l'article d'Eduardo Viveiros de Castro, "Perspectival Anthropology and the Method of Controlled Equivocation" (2004b) - l'une des références classiques du renouveau contemporain des oppositions radicales «nous »/«eux» par lesquelles j'ai ouvert mon propos.

Cet article de Viveiros de Castro s'oppose explicitement au célèbre appel fonctionnaliste de Fred Eggan à la " comparaison contrôlée ", c'est-à-dire à des comparaisons prudentes et partielles de sociétés similaires dans des espaces limités. Mais il utilise Eggan comme tremplin pour un exercice tout à fait différent : tenter d'esquisser un manifeste épistémologique pour une anthropologie qui soit une mise à nu radicale de la différence entre les mondes conceptuels de l'indigène et de l'anthropologue. En faisant cela Viveiros de Castro établit une distinction entre ce que j'appelle ici la comparaison latérale ("comparaison entre différentes instanciations spatiales ou temporelles d'une forme socio-structurelle donnée ", Viveiros de Castro 2004b : 4) et la démarche frontale qu'il qualifie de «comparaison traductive»:

"[...] la traduction des concepts pratiques et discursifs de l' "indigène" dans les termes de l'appareil conceptuel de l'anthropologie. Je fais allusion à ce genre de comparaison, le plus souvent implicite ou automatique (et ainsi incontrôlée), qui inclut nécessairement le discours de l'anthropologue comme un de ses termes, et qui se met en place dès le début du travail de terrain, sinon bien avant » (Ibid.). 
Cette distinction fait écho à celle d'Evans-Pritchard, mais avec deux différences importantes et riches d'enseignement. Premièrement, la nature de l'hinterland et, avec elle, la manière dont la comparaison frontale fonctionne comme une heuristique ont subtilement changé. Deuxièmement, l'ordre et la priorité des comparaisons frontale et latérale se sont inversés.

Le premier changement a été longuement discuté ailleurs. Sur certains points essentiels, le «nous " d'Evans-Pritchard et le «nous » de Viveiros de Castro ne sont plus les mêmes. Ce qui les sépare relève entre autres d'une série de bouleversements politiques, historiques et épistémiques. L'hinterland comme procédé heuristique a été reconfiguré et adapté à un nouveau terrain politique et épistémique. Il en résulte que la comparaison frontale elle-même a changé de nature, jusqu'à un certain point.

Les comparaisons frontales d'Evans-Pritchard se fondaient sur et contribuaient à renforcer l'idée d'un monde où l'existence d'entités culturelles ou civilisationnelles largement distinctes était un fait acquis. C'est ce qui a été défini comme un paysage "pluraliste " (Holbraad \& Pedersen 2009) : un paysage inscrit dans le langage de la partie et du tout, des «ensembles culturels cohérents à l'intérieur et différents de ce qui est ailleurs " (Mol 2002). Les comparaisons frontales d'Evans-Pritchard reposaient également sur le fait que l'anthropologie comme projet appartenait clairement à l'un d'eux ${ }^{8}$. Dans ce contexte, la comparaison frontale venait apporter un éclairage par la double démarche consistant à rendre l'étrange familier et le familier étrange. Cette dynamique n'est certes pas anodine. Elle implique un certain nombre de présupposés quant à savoir de qui parle l'anthropologie et à qui elle s'adresse. Comme Clifford Geertz l'a remarqué, les anthropologues comme Evans-Pritchard écrivaient comme si « leurs objets et leur public étaient non seulement séparables mais moralement distincts, qu'il fallait décrire les premiers mais pas s'adresser à eux, informer le deuxième mais pas l'impliquer» (1996 [1988] : 132).

Aucun anthropologue écrivant aujourd'hui ne peut agir ainsi en conscience. Comment, dès lors, devons-nous comprendre la persistance des comparaisons frontales? La réponse est pragmatique et politique : l'un des avantages de la comparaison frontale comme heuristique - sa capacité à susciter un réexamen critique de "nos propres catégories" (ou, plus largement, de nos propres politiques, de nos propres engagements) était trop précieux pour être abandonné. C'est pourquoi, en même temps que la dimension politiquement et épistémiquement intenable de

8. Par exemple, l'avant-propos d'Evans-Pritchard à l'étude de l'Espagne rurale par Julian PittRivers (1954) réfléchit sur les défis et les promesses de l'anthropologie en ce qu'elle essaye d'étudier "notre propre civilisation ". 
l'«altérisation" était dénoncée (Said 2003 [1978]), le frontal était transformé en "critique culturelle" (Marcus \& Fischer 1999 [1986]). Une bonne partie de la remise en question politique et épistémologique que traverse l'anthropologie depuis les années 1980 portait sur cette quadrature du cercle : comment conserver un potentiel critique suffisant pour remettre en question "nos propres présupposés", et ne tenir pour acquis ni l'image naïve d'un monde divisé en cultures distinctes, ni le privilège épistémique accordé à l'anthropologie pour les décrire?

Si l'on admet que la comparaison frontale est une ressource précieuse, comment l'envisager à nouveau dans un environnement conceptuel postpluraliste, où la référence à des unités culturelles standards (etc.) n'est plus explicitement défendable? Une réponse - de loin la plus courante a été simplement de poursuivre comme si de rien n'était, sans trop se soucier des fondements épistémiques d'une telle pratique - en considérant les théories pluralistes comme "heuristiques» au sens faible et vague.

Il y a eu pourtant deux types d'approches influentes et effectives, par lesquelles la comparaison frontale a été explicitement redéfinie comme heuristique (au sens fort et technique) pour un monde théorique postpluraliste. La première approche consista à intensifier à la fois l'aspect polémique de la comparaison frontale et sa dynamique d'auto-distanciation. L'hinterland, auquel on confronte un nouvel objet, en vint à renvoyer non pas à un ensemble de présupposés (culturels et propres à la discipline) pouvant servir de grille de lecture perfectible pour l'ethnographie, mais à une position conceptuelle politiquement dominante, dont on suppose qu'elle serait largement partagée dans le milieu de l'auteur, mais à laquelle l'auteur lui-même serait par définition hostile : les conceptions «libérales » occidentales, par exemple, ou les visions eurocentriques de la "modernité ». La référence au "nous » repose alors sur le postulat empirique qu'une telle position est en effet partagée par un grand nombre de personnes et qu'il en résulte des effets concrets sur le monde, auxquels le travail du chercheur apporterait un contrepoint critique. Ainsi, par exemple, Chakrabarty explique sa référence constante à l'« Europe » et l' "Inde » :

" "Europe" et "Inde" sont ici traités comme des termes hyperréels, en ce sens qu'ils se rapportent à des figures imaginaires dont les référents géographiques conservent une part d'indétermination [...]. D'emblée, les chercheurs libéraux s'insurgeront, en disant que l'idée d'une "Europe" homogène et incontestée ne résiste pas à l'analyse. C'est parfaitement exact, mais de même que le phénomène de l'orientalisme n'a pas disparu parce que certains en ont désormais une appréhension critique, de même une certaine version de l' "Europe", réifiée et célébrée dans le monde phénoménal des rapports de pouvoir quotidiens en tant que scène de naissance de la modernité, continue de dominer le discours de l'histoire. L'analyse est impuissante à l'éradiquer " (2009 [2000] : 68). 
L'autre approche - plus rare quoiqu'amplement discutée récemment sous l'étiquette de "tournant ontologique» - a été de transformer l'hinterland en un postulat s'auto-justifiant: un procédé qui permet à la comparaison frontale d'établir de façon récursive la différence même sur laquelle elle repose. La clé de cette démarche était déjà présente dans ce que j'ai décrit plus haut comme la dualité de l'hinterland: sa double référence à un milieu culturel et propre à la discipline. Initié par Roy Wagner (1981 [1975]) et Marilyn Strathern (1988), popularisé et affiné depuis par d'autres (Viveiros de Castro 2004b ; Holbraad 2012; Henare, Holbraad \& Wastell 2007), ce courant consiste à effacer la distinction entre ces deux aspects de l'hinterland en établissant une comparaison directement entre les catégories analytiques de l'anthropologue (culture, société, individu, agencéité, etc.) et celles des personnes étudiées. Dans cette rencontre entre les catégories de l'observateur et celles des observés, apparaît un nouveau fondement, incontestable, de la réalité de la différence, un nouveau minimum inconcussum quid. "Les connaissances propres à la discipline" s'avèrent n'être, après tout, qu'un aspect des "catégories conceptuelles de [notre] propre culture». À l'inverse, et en même temps, l'existence de "notre propre culture "s'incarne minimalement dans "les connaissances propres à la discipline", ou même, simplement, dans les catégories sélectionnées pour être examinées par cet anthropologue particulier à ce moment particulier ${ }^{9}$.

La façon dont Marilyn Strathern, par exemple, met en garde contre son usage du terme " occidental » dans The Gender of the Gift, met en évidence les effets de cette démarche :

"Je voudrais faire ressortir un certain nombre d'idées sur la nature de la vie sociale en Mélanésie en les confrontant à des idées présentées comme l'orthodoxie occidentale. Mon étude ne requiert pas que ces dernières soient orthodoxes pour tous les penseurs occidentaux; la place qu'elles tiennent est celle d'une position stratégique interne à la structure de la présente étude» (1988: 12).

L'étude ne nécessite pas l'uniformité d'une tradition occidentale parce que l'existence même de cette étude et des catégories qu'elle mobilise (les notions classiques de personne, d'agencéité ou de société) suffit à prouver l'existence d'un vaste hinterland occidental avec lequel l'ethnographie peut être mise en contraste.

9. Pour une façon différente d'interpréter la reformulation de la comparaison postpluraliste dans le travail de Marilyn Strathern, cf. Martin Holbraad \& Morten Axel Petersen (2009). Si je m'inspire de leur présentation, la mienne diverge sur plusieurs points. En particulier, la notion d' "abstention" pour désigner le type singulier de concept-chose produit par les comparaisons de Strathern me semble assez obscure, une manière de pointer un paradoxe plutôt que de l'éclairer. 
Lillustration la plus évidente de cette approche consistant à rendre l'hinterland auto-suffisant vient de Tim Ingold. Après avoir montré les limites de la comparaison frontale telle qu'elle est couramment pratiquée en anthropologie (cf. supra), Ingold poursuit:

"Pour ceux d'entre nous qui se définissent comme universitaires ou intellectuels, il y a une bonne raison à ce que nous ne puissions échapper à l' "Occident", ni éviter les affres de la modernité. C'est que notre activité même, quand nous pensons et nous écrivons, est sous-tendue par une croyance en la valeur absolue de l'enquête disciplinée, rationnelle. Dans ce livre, c'est à cette croyance que les termes "occidental" et "moderne" renvoient. Et quelles que soient nos objections face aux dichotomies qu'ils engendrent, entre humanité et nature, intelligence et instinct, mental et physique (etc.), l'art de la discussion critique sur ces sujets est précisément toute l'affaire de l' "Occident". Car, tout bien considéré, il n'y a rien de plus "occidental", ni de plus “moderne" que d'écrire un livre académique tel que celui-ci” (2000:6-7).

Ces deux manières de réutiliser l'heuristique de la comparaison frontale - par la critique politique ou l'exemplification épistémique du soi - ne sont pas mutuellement exclusives. Parfois, elles sont explicitement combinées dans l'élaboration d'un manifeste pour une « révolution conceptuelle permanente " comme acte intrinsèquement politique (Holbraad, Pederson \& Viveiros de Castro 2014). Fondamentalement, elles partagent également, en l'intensifiant, la logique de distanciation du soi que j’ai déjà désignée comme étant constitutive de l'hinterland d'Evans-Pritchard: alors que l'hinterland fait appel à une terre partagée, que les lecteurs reconnaittront facilement, la référence même à cette terre commune est la démarche par laquelle l'anthropologue s'en distancie lui-même. Dès le moment où le terme "culture ", par exemple, devient visible comme "notre" catégorie analytique, elle ne fait plus directement partie de la boîte à outils de l'auteur.

Ces deux manières de revitaliser l'heuristique de la comparaison frontale évitent d'avoir à définir l'hinterland à partir des vieux termes pluralistes de culture ou de civilisation. Il en résulte que la comparaison frontale n'offre aucune garantie quant aux limites ou à la configuration de l'hinterland auquel elle renvoie. À vrai dire, elle ne l'a jamais fait. Personne ne consulte les écrits d'Evans-Pritchard sur la sorcellerie azande, ni même La Pensée sauvage de Lévi-Strauss (1962) pour y trouver une caractérisation stricte des présupposés scientifiques occidentaux (Salmon 2013). Demander cela à la comparaison frontale, c'est demander à tort. La comparaison frontale n'est pas, par définition, une ethnographie de l'hinterland. En fait, une caractéristique de l'heuristique est que l'hinterland mobilisé est nécessairement exclu de ce type d'analyse approfondie (Viveiros de Castro 2011 ; Candea 2011). Une ethnographie de l'hinterland - une anthropologie "chez soi»- est un exercice tout à fait différent (Strathern 1987). 
Un aspect plus problématique de la comparaison frontale ainsi renouvelée est qu'elle risque de faire coïncider systématiquement le «sens commun" occidental avec certaines théories adverses. Comme l'écrivait Bas Van Fraassen, «chaque philosophe ou presque commence par expliquer qu'il s'oppose à la conception "dominante" ou "convenue" et présente la sienne comme révolutionnaire" (1980:4); les anthropologues ne procèdent pas autrement à cet égard. Il n'y aurait rien d'intrinsèquement gênant là-dedans, si les autres engagements (épistémologiques, politiques, etc.) des anthropologues ne convergeaient par ailleurs. Par conséquent, les hinterlands qu'ils esquissent - comme repoussoirs - sont souvent d'inspirations assez semblables : cartésienne, kantienne, néolibérale, individualiste, etc. Aucune opposition frontale, à ma connaissance, ne se donne pour but de "déstabiliser nos présupposés deleuziens occidentaux ", par exemple. Si les hinterlands sont similaires, cela produit une tendance à mettre les ethnographies au même niveau. À mon sens, c'est là qu'est la clé de ce problème souvent constaté avec la comparaison frontale - le tournant ontologique ne faisant pas exception : la similarité surprenante qu'elle tend paradoxalement à produire à partir de l'analyse d'entités ethnographiques radicalement distinctes. Étant donné que le but de la comparaison frontale est la perturbation conceptuelle, il s'agit d'une défaillance potentiellement sérieuse.

\section{La persistance du latéral}

Pour résumer, la comparaison frontale en tant qu'heuristique s'est donc relevée, quoique transformée, du discrédit épistémologique dans lequel elle était tombée dans les années 1980. En renaissant de ses cendres pour devenir critique politique, innovation conceptuelle radicale ou, dans certains cas, les deux, elle a survécu et est même passée au premier plan de l'agenda de notre discipline.

Cette histoire en elle-même n'est pas nouvelle, elle a déjà été racontée auparavant, encore que peut-être pas tout à fait en ces termes. Ce sur quoi je voudrais attirer l'attention est plutôt ce qui fait défaut, à savoir le fait qu'aucune récupération épistémologique semblable n'ait été faite, ni même tentée, pour la comparaison latérale à l'échelle de la discipline. La comparaison latérale a pourtant été tout autant affectée, de jure, par la crise épistémique des années 1980. Tout comme la comparaison frontale, elle continue à être au fondement du travail anthropologique - par définition, nous ne pouvons pas nous en passer. Mais personne n’a cru bon rédiger un nouveau manifeste sur son importance ou sa pertinence ${ }^{10}$.

10. Cf. cependant Morten Axel Pedersen \& Morten Nielsen (2013) ou Marilyn Strathern (2004). 
Cela est révélateur d'un changement plus profond en termes d'intérêts et d'objectifs. Pour Evans-Pritchard et la plupart de ses disciples, la comparaison frontale entre "leurs" concepts et les "nôtres" n'était qu'une première étape dans l'activité de production du savoir anthropologique. Une étape cruciale et décisive bien sûr, mais, en elle-même, purement "littéraire ». Pour Viveiros de Castro comme pour beaucoup d'anthropologues aujourd'hui, la situation est inversée. La comparaison latérale n'est qu'un type d'investigation anthropologique optionnel ${ }^{11}$, alors que la comparaison frontale est devenue " une règle constitutive de la discipline", la définition même de l'anthropologie :

«Le problème essentiel de l'anthropologie consiste moins à déterminer quelles relations sociales constituent son objet qu’à demander ce que son objet constitue comme relation sociale - ce qu'est une relation sociale dans les termes de son objet, ou mieux encore, dans les termes qui émergent de la relation (relation sociale, naturellement) entre l' "anthropologue" et l' "indigène" " (Viveiros de Castro 2004b : 4).

Ainsi Viveiros de Castro (à la suite d'Asad) inverse les propos d'EvansPritchard: "la comparaison est au service de la traduction et non l'inverse " (Ibid.: 5 , italiques dans le texte). Si on la considère attentivement toutefois, cette inversion est également une forme d'englobement. Progressivement - et de façon un peu déroutante -, la "comparaison" dans le texte est largement confondue avec la "traduction». La comparaison frontale (traduction) est devenue la comparaison tout court. La comparaison latérale (l'examen de cas côte à côte) disparaît entièrement des préoccupations, alors que le débat sur la comparaison frontale s'intensifie et s'élargit.

Rien de nouveau en cela ${ }^{12}$. Pour les raisons évoquées dans la section précédente, la comparaison frontale a obtenu la part du lion de l'attention conceptuelle et méthodologique en anthropologie, depuis au moins les années 1970. "Traduction ", "altérisation ", "incommensurabilité ", "observation participante ", "réduction épistémique ", " équivocation", " récursivité » ou "symétrisation » - à travers ces thèmes et d'autres encore, la possibilité, les méthodes et les effets des confrontations entre "nous » et " eux" ont été parmi les sujets de réflexion les plus constants pour les épistémologues de l'anthropologie (y compris ceux qui se disent aujourd'hui "ontologistes»). Les réflexions explicites sur la comparaison latérale, en revanche, ont été fort rares durant les dernières décennies.

11. "Ce type de comparaison n'est qu'un modèle régulateur - et d'autres formes d'investigation anthropologiques existent » (Viveiros de Castro 2004b : 4).

12. «Le problème de la comparaison devient le problème de la traduction (Hobart) et toute notre analyse est en dernière instance comparative en ceci que nous devons traduire pour être capable de décrire (Overing)» (Holý $1987:$ 5). 
Comme je l'ai remarqué plus haut, les débuts de ce changement ont été correctement diagnostiqués par ceux qui cherchaient à caractériser le passage du "positivisme" à l' "interprétivisme" en anthropologie, dans les années 1970 (Holý, ed. 1987). Je voudrais simplement rectifier la version de Holý en faisant remarquer que ceux qu'il décrit comme "positivistes» se préoccupaient bel et bien des questions "frontales » d'administration de la preuve, d'interprétation, de traduction des catégories indigènes en catégories analytiques et leur cortège de problèmes associés (Lewis 1955 : 268). C'est juste que, dans l'ensemble, ils les traitaient - ainsi qu'Evans-Pritchard le faisait lui-même - comme des questions de technique et de compétence individuelles, comme une sorte de savoirfaire professionnel que tout bon anthropologue se devait d'apprendre à maitriser. Ces savoir-faire et ces techniques pouvaient être abordés dans un cadre pédagogique, sous la forme de conseils prodigués par les professeurs à leurs étudiants par exemple, mais elles ne faisaient pas l'objet de discussions approfondies et explicites au sein de publications académiques, à l'instar des problèmes « conceptuels" plus élaborés de comparaison latérale. Il se peut également que cette "connaissance tacite» comme idéal régulateur ait été une façon, pour les anthropologues, de faire face à l'embarras causé par l'inévitable recours à une méthode irréductiblement "subjective » dans une discipline qui aspirait à l'objectivité. Quoi qu'il en soit, alors que la comparaison latérale était supposée requérir un ensemble partagé de règles opérationnelles qui suscitaient de nombreuses discussions et débats académiques, la comparaison frontale était globalement abandonnée à l'initiative individuelle, voire au talent des spécialistes, tout en considérant qu'elle répondait à des normes implicites.

La situation a été essentiellement inversée avec l'interprétivisme, et cette inversion s'est maintenue bien après son déclin. Tandis que les anthropologues ont aujourd'hui pour préoccupation majeure les implications épistémiques, éthiques et politiques de la comparaison frontale, élaborant des principes et montrant pourquoi et comment la pratiquer, la comparaison latérale se maintient comme pratique de routine, le plus souvent sans surveillance épistémologique.

Dans leurs échanges et dans leurs publications, les anthropologues établissent constamment des comparaisons latérales entre différents moments de leur expérience de terrain, entre différents sites sur lesquels ils ont travaillé, entre différentes études de cas issues de leur aire de spécialisation géographique ou thématique, ou au-delà. Mais l'on envisage rarement que cela exige une élaboration plus large de normes partagées sur qu'est-ce qui est «comparable» et comment. 
En somme, un peu comme pour la comparaison frontale dans les années 1940 et 1950, la comparaison latérale est devenue principalement aujourd'hui un espace d'initiative individuelle, régi par un savoir tacite et par des compétences le plus souvent implicites. Une bonne part de ce qui fait un «bon " anthropologue implique de faire preuve d'originalité et de talent en recourant à des juxtapositions de cas astucieuses et inattendues, alors que faire preuve d'une grande maîtrise intellectuelle s'entend comme la capacité à avoir une vue d'ensemble de cas comparables de façon à délimiter un espace à l'intérieur duquel son propre cas pourra avoir un effet transformateur (et non pas simplement "remplir un vide", comme on le rappelle constamment aux étudiants).

Ici également, l'embarras peut jouer un rôle. Après tout, comme je l'ai remarqué plus tôt, l'anthropologie d'aujourd'hui a rejeté un grand nombre des principes fondamentaux qui rendaient possible la comparaison latérale de jure pour nos prédécesseurs : la notion d' "unités " comparables, l'idée de catégories généralisables stables et l'image de l'anthropologue comme acteur privilégié de la comparaison des mondes autres. La plupart des discussions sur la comparaison frontale tournent précisément autour des manières de dépasser ces visions limitées - en étant dialogiques, par exemple, ou en aspirant à la révolution permanente de nos mondes conceptuels. Mais cela laisse la comparaison latérale à la porte. Le fait que nous continuions pourtant à comparer latéralement, à bien y penser, devrait être aussi choquant pour les sensibilités contemporaines que la nature irréductiblement subjective des connaissances acquises sur le terrain l'était pour un auteur objectiviste du milieu du XXe siècle.

Les recherches de Viveiros de Castro sur le perspectivisme amazonien illustrent précisément cette dynamique. Au cœur de son travail, se trouve une confrontation majeure entre les ontologies amazoniennes et les caractéristiques des "nôtres" (Viveiros de Castro 1998, 2004a, 2011). Ce contraste frontal est le point focal d'une grande partie de ses recherches, ainsi que l'objet d'une attention méthodologique et conceptuelle considérable, comme dans l'article évoqué plus haut. De telles comparaisons frontales visent à bouleverser "nos " catégories analytiques, à remettre en question les conceptions de la culture et de la différence, à réinventer l'anthropologie comme lieu de révolution conceptuelle permanente.

Or, en établissant ce contraste entre le naturalisme euro-américain et le multi-naturalisme amazonien, Viveiros de Castro s'appuie largement sur les comparaisons latérales entre les différents cas amazoniens, s’inspirant de son propre travail et de celui des autres pour dégager des schémas communs propres aux institutions et aux pratiques, réinterpréter les 
différences observées ou établir des analogies et des continuités à partir de différents domaines de pratiques sociales. Dans leur ensemble, ces approches comparatives appartiennent à une forme d'anthropologie classique facilement identifiable et, contrairement à la comparaison frontale, les comparaisons latérales "à l'intérieur de l'aire régionale » sont fournies sans trop de commentaires explicites ni de remises en question méthodologiques. Elles forment le matériau de base, usuel, à partir duquel l'argumentation principale est construite, et elles ne sont pas présentées comme étant source de difficultés majeures, ni, en tant que telles, d'inspiration décisive.

En d'autres termes, alors que les comparaisons frontales de Viveiros de Castro aspirent à remettre profondément en question et démonter les fondements mêmes du savoir anthropologique, les éléments qui les constituent (du côté amazonien du moins) sont des comparaisons latérales s'inscrivant dans la plus pure tradition anthropologique. D'un point de vue méthodologique, ces comparaisons latérales évoquent et renforcent cette image rassurante qu'on en avait au milieu du $\mathrm{XX}^{\mathrm{e}}$ siècle $^{13}$, à savoir : un ensemble stable d'études similaires sur des groupes sociaux distincts au sein d'une même région; des études produites par un ensemble d'observateurs-participants tous membres de la même communauté épistémique; des études qui décrivent des pratiques, des cosmologies et des institutions pouvant être comparées et mises en contraste afin d'identifier des régularités ou des différences plus générales, tout en continuant à tester et modifier les hypothèses à propos de ces schémas.

\section{Une étude de cas}

Dans la dernière partie de cet article, je vais tenter d'approfondir ma caractérisation de la persistance et de la productivité de la comparaison latérale dans l'anthropologie contemporaine, à partir de la lecture attentive d'un article de Carlos Fausto, qui illustre de façon remarquablement claire, à la fois les différentes sortes de démarches latérales que les anthropologues mobilisent habituellement, et les présupposés épistémologiques divers et souvent implicites qui les sous-tendent.

13. Bien sûr, les comparaisons de Viveiros de Castro proviennent d'une tradition structuraliste qui s'écarte sur des points fondamentaux du type de comparatisme de l'anthropologie du milieu du siècle par lequel j’ai commencé ma présentation (Salmon 2013). Néanmoins, même si je n'ai pas la place de développer ce problème ici, je soutiendrai que les fondements de ma distinction frontal/latéral vont au-delà des différences que l'on établit souvent entre les formes structuralistes et fonctionnalistes de la comparaison. 
L'article de Carlos Fausto, «Feasting on People» (2007), constitue une tentative audacieuse pour penser comparativement dans plusieurs directions en même temps. S'appuyant sur une série d'ethnographies du chamanisme d'Amazonie et d'Amérique du Nord, Fausto cherche à expliquer ce paradoxe: comment, dans des contextes animistes où les animaux sont aussi des personnes, la consommation d'une proie peut-elle être différenciée d'un acte de cannibalisme? Pour Fausto, une des réponses possibles s'appuie sur une représentation de la chasse comme forme d'échange de dons non violent, au cours duquel ce qui est consommé dans l'animal n'est pas son âme anthropomorphe, mais simplement son corps, donné librement au chasseur. L'acte cannibale est ainsi éludé.

Selon Fausto, cette interprétation, basée principalement sur des ethnographies de sociétés animistes d'Amérique du Nord, ne s'applique pas très bien aux sociétés animistes d'Amazonie ${ }^{14}$. Il trouve en effet chez ces dernières une dynamique différente, selon laquelle la personne (qu'elle soit humaine ou non humaine) est composée d'une partie active (prédateur) et d'une partie passive (proie), qui sont séparées l'une de l'autre au moment de leur consommation. Le cannibalisme consiste en l'ingestion de la partie active de la proie. Cette pratique, typique du chamanisme par exemple, est un vecteur dangereux de transformation mutuelle, par laquelle le consommateur risque d'être capturé par la proie. La consommation à des fins culinaires, en revanche, nécessite l'accomplissement d'opérations spécifiques visant à désubjectiver la proie et à s'assurer ainsi que ce sont bien ses parties passives qui sont consommées. Ces opérations rendent possible la commensalité et la production de liens de parenté. D'après cette redéfinition du cannibalisme, l'acte d'anthropophagie rituelle ne relève plus du cannibalisme, mais simplement de la consommation d'un corps désubjectivé qui se trouve être humain - configuration particulièrement poussée dans laquelle la parenté est redéfinie à partir de la commensalité.

L'analyse de Fausto s'ouvre sur une comparaison frontale, mais s'en écarte rapidement. Dans le premier paragraphe, il remarque que, depuis Darwin, la pensée occidentale a cherché à comprendre comment le fait de manger de la viande a pu façonner la socialité et l'identité des hommes modernes. Pour preuve de ce qu'il avance, il s'appuie sur les travaux des anthropologues et des archéologues évolutionnistes qui ont tenté de relier la consommation de viande aux origines de la guerre, de l'organisation

14. Fausto établit également un contraste avec des exemples sibériens, qu'il décrit comme constituant un point intermédiaire entre les cas amazoniens et nord-américains. Je laisse cela de côté par souci de simplicité. 
sociale, des relations entre les genres et du partage. Après avoir esquissé cet hinterland, la suite de l'article va consister à s'y opposer :

" Dans cet article, je veux mettre en perspective cet ensemble d'interprétations à partir d'une tradition de pensée différente, qui s'est développée dans les Amériques des milliers d'années avant l'arrivée des Européens modernes et qui est toujours très vivante aujourd'hui. Cette tradition a émergé à partir de présupposés ontologiques tout à fait distincts, conduisant à des conceptualisations de la relation entre la prédation et la consommation de nourriture et à des pratiques sociales qui sont tout à fait différentes. Les ontologies amérindiennes ne sont pas fondées sur le dualisme entre nature et culture (ou entre sujet et objet) qui joue un rôle fondateur dans la tradition occidentale moderne» (Fausto 2007 : 497).

Cette approche porte bien la marque de la comparaison postpluraliste évoquée plus haut: la preuve de l'altérité radicale de l'Occident est recherchée dans un ensemble de discours académiques très proches (ici, ceux de l'anthropologie évolutionniste), sans pour autant les recouper avec ceux de l'anthropologue lui-même. Le contraste est ainsi établi, simultanément, entre deux "traditions de pensée » et entre un ensemble de savoirs académiques auquel on oppose un objet nouveau et stimulant.

Traitées séparément, aucune de ces deux démarches ne tient vraiment debout. Si le but est de mettre deux traditions en contraste, alors on a inévitablement l'impression que la caractérisation de la tradition occidentale en référence à ces présupposés évolutionnistes spécifiques ne résiste pas longtemps à l'examen. Qu'en est-il de toutes les autres manières de relier la consommation de viande et la socialité en Occident ? Pourquoi choisir cet exemple en particulier? Si le but est de recourir au cas amazonien pour interroger les présupposés d'une communauté spécifique de chercheurs sur le rôle de la consommation de viande dans l'évolution, alors, encore une fois, on ne voit pas très bien comment cela pourrait fonctionner, puisque le matériau amazonien ne peut avoir valeur de preuve selon les critères de la littérature invoquée ici. En toute évidence, cette discussion sur la façon dont les Amazoniens "comprennent" la prédation et la vie sociale ne saurait avoir le pouvoir de remettre en question les théories évolutionnistes.

En revanche, si on les associe, ces deux démarches se complètent et se renforcent, comme les deux côtés d'une même argumentation. Côté face, l'objection, selon laquelle le matériau amazonien n'est qu'un exemple d'une autre perspective culturelle laissant intacts les fondements de l'évolution, est contrée par le fait même de traiter la perspective évolutionniste comme n'étant qu'un exemple parmi d'autres de la pensée occidentale. Côté pile, l'idée de prendre la littérature évolutionniste comme un concentré de la pensée occidentale est à son tour rendue 
pertinente justement par le fait que le lecteur a pu concevoir l'objection ci-dessus. Évolutionniste ou pas, il vient de prouver qu'il partage les présupposés épistémologiques de ce qu'on nomme le "naturalisme».

Remarquez que c'est moi qui vois un projet nettement heuristique dans cette première page. À aucun moment cette intention postpluraliste n'est explicitée. On pourrait tout aussi bien lire cette mise en contraste dans les termes classiques d'un face-à-face pluraliste entre civilisations distinctes, à la* Evans-Pritchard. Il est intéressant de constater que cette question épistémologique de fond s'avère finalement avoir peu d'impact. Qu'il soit pluraliste ou postpluraliste, l'effet de l'attaque frontale d'ouverture sur l'ensemble de l'argumentation est évident: le sujet traité est mis en perspective par cette entrée en matière* qui neutralise le réflexe, chez certains lecteurs, de vouloir trouver des explications fonctionnalistes ou évolutionnistes aux pratiques amazoniennes de consommation de viande.

Toujours est-il que l'essentiel de cet article consiste en comparaisons latérales plutôt que frontales. Elles se présentent sous trois formes, chacune s'appuyant sur des présupposés épistémologiques tout à fait différents - sans que cela soit peu ou prou reconnu explicitement. La première est la démarche classique de construction d'un "modèle théorique" établi à partir des points communs trouvés au sein d'un échantillon varié et soigneusement choisi d'ethnographies de la région, auxquelles ce modèle est supposé s'appliquer ( «certains exemples pris dans toutes les grandes familles linguistiques des basses terres d'Amérique du Sud» [Fausto 2007 : 498]). Le but est d'identifier un ensemble de "principes" communs qui sous-tendent la "variation culturelle " à l'intérieur de cette aire régionale. Une «hypothèse » que les autres ethnographes spécialistes de cette même région devraient pouvoir reconnaitre dans leurs propres cas d'étude. Nous avons là, n'en déplaise à Viveiros de Castro, un exemple presque parfait du genre de "comparaison contrôlée ", qu'Eggan aurait reconnu comme telle et probablement admirée ${ }^{15}$.

* En français dans la version originale $[N d t]$.

15. "Même de nos jours, on peut observer une variation culturelle considérable à travers la région, et un modèle amazonien de la relation entre la prédation et la consommation de nourriture peut sembler excessivement généralisateur. Je voudrais décrire le fondement sur lequel une partie de cette variation émerge et démontrer la solidité ethnographique de mon modèle à travers certains exemples pris dans toutes les grandes familles linguistiques de l'Amérique du Sud des basses terres. Je ne prétends pas que ce modèle s'applique à tous ces groupes humains de la même façon ou dans les mêmes domaines. Néanmoins, la couverture ethnographique est assez étendue et diverse pour rendre plausible mon hypothèse selon laquelle nous avons affaire à un substrat avec de larges implications dans la vie quotidienne des peuples amazoniens. En tant que modèle, il est certainement abstrait, mais cela ne veut pas dire qu'il n'est pas enraciné dans le tissu de l'expérience concrète quotidienne des peuples indigènes. J'espère que d'autres ethnographes reconnaîtront que les principes énoncés ici opèrent dans le contexte de leur propre terrain et sont incarnés dans .../... 
Les observations de Fausto sur la nature et les limites de la comparaison latérale sont exemplaires. Les réserves qu'il émet portent la marque d'une comparaison contrôlée considérée comme heuristique : elle est, bien sûr, très abstraite et ne peut rendre compte de la réalité expérientielle des pratiques que seule une description ethnographiquement fondée peut permettre de restituer. Les principes qu'elle dégage sont susceptibles de s'appliquer de différentes façons et à différents endroits. Elle s'adresse à et est contrôlée par d'autres ethnographes de la même région. Elle sera retenue ou rejetée selon que ces autres ethnographes la jugeront pertinente pour leur propre cas d'étude.

La deuxième forme de comparaison latérale établie dans l'article de Fausto concerne les ethnographies des sociétés animistes en dehors de l'aire amazonienne. Ici, en revanche, le but est de mettre en évidence les différences au sein d'un domaine élargi :

"Les ontologies amazoniennes font partie de ce que j'appellerais la tradition sibérochamanique, qui a une unité historique à part entière. Mon argumentation se développe à partir d'un contraste interne avec les chasseurs-cueilleurs de la région subarctique, locuteurs des langues algonquiennes et athapascanes, qui vinrent à représenter dans sa forme la plus pure la conversion de la chasse en une relation positive de don et de partage. Ce modèle est basé sur le remplacement de la prédation par le don" (Ibid.: 498).

Ces deux types de comparaison latérale opèrent symétriquement dans deux directions opposées pour souligner la spécificité de l'Amazonie en tant qu'espace ethnographique. En ce sens, ce sont des comparaisons typiquement pluralistes, qui indiquent des «ensembles culturels, cohérents à l'intérieur et différents de ce qui existe ailleurs» (Mol 2002: 80). Or, une incertitude significative se glisse dans cette deuxième approche, qui était absente de la première. Ici, Fausto émet un doute sur le matériau nord-américain :

"Tandis que dans la région subarctique le don règne en maître, la prédation est le schéma le plus productif en Amazonie. Il est difficile de déterminer si ce contraste est entièrement empirique ou s'il résulte également des différences d'approche entre chercheurs dans les deux aires régionales» (2007: 500).

L'effet potentiellement néfaste de cette incertitude est néanmoins rapidement contré et neutralisé :

«Dans notre approche, le contraste avec le cas nord-américain, qu'il soit littéral ou littéraire, nous permet de mettre en évidence un aspect qui est resté largement inexploré ailleurs et qui semble être central dans la forêt tropicale : que la prédation est un vecteur transspéciste de socialité » (Ibid.).

[Suite de la note 15] l'expérience quotidienne. Je ne prétends pas donner une restitution phénoménologique du modèle, ce qui, je crois, ne peut être fait sérieusement que dans un registre plus ethnographique» (Fausto $2007: 498$ ). 
À première vue, ce manque d'intérêt pour la différence entre le "littéral " et le "littéraire» ne cadre pas avec la justification empirique bien soignée du modèle amazonien dans le premier type de comparaison. Pourquoi prendre tant de précautions si le littéral et le littéraire sont largement interchangeables? On s'interroge aussi sur les intentions de Fausto quand il semble mettre en doute des analyses particulières du matériau nord-américain, quand il propose des redéfinitions plus générales de concepts tels que le cannibalisme, ou quand il prétend "s'écarter" du dualisme classique corps/esprit ${ }^{16}$ d'une manière qui ressemble beaucoup à une critique de l'état de l'art du moment. L'un des commentateurs de l'article l'ayant interpellé à ce sujet ${ }^{17}$, Fausto retombe dans l'ambiguïté :

" [Vaté] me reproche de proposer une interprétation différente d'une certaine dimension du travail de Brightman. Qu'il soit bien clair que je ne "l'accuse pas de mal comprendre son propre matériel ethnographique". Je propose simplement une interprétation différente de ce que représente le cannibalisme en Amazonie et dans la forêt boréale. Quand on veut faire un travail comparatif, on doit aborder les données des collègues de façon critique» (Ibid. : 521).

Mais, si l'intention est juste d'avancer qu'il n'en va pas de même en Amazonie, on peut très bien imaginer Virginie Vaté répliquer : en quoi est-ce une révision critique des données d'un collègue, ou même une interprétation différente de son travail ? Et si le but est d'identifier une dynamique régionale, alors comment Fausto peut-il affirmer en même temps qu'il ne s'intéresse pas au fait qu'il existe des exemples de dynamique du don en Amazonie également ${ }^{18}$ ?

$\mathrm{Si}$, compte tenu de l'orientation générale de cet article, nous évitions le réflexe critique du «tout ou rien » et considérions ce type de comparaison latérale comme une heuristique, nous dirions, non pas qu'elle est moins

16. "Dans le cadre de ce paradigme, la chasse apparaît comme un partage entre humains et animaux et s'oppose ainsi aux relations belliqueuses entre humains. Mais quelle est l'extension de ce paradigme? S'applique-t-il à tous les systèmes animistes ou à tous les chasseurs-cueilleurs?" (Fausto 2007 : 498). "La réponse de Brightman ne dépend pas seulement du postulat de la possibilité d'une distinction entre un corps zoomorphique et une âme anthropomorphique, mais également de l'adoption de l'idée que le cannibalisme est la consommation du même. Dans cet article je me suis écarté de cette notion, en essayant de redéfinir le cannibalisme comme la consommation de la partie active de l'autre. J'ai essayé de montrer que même si la formulation de Brightman en termes de dualisme corps/esprit peut être commode, elle ne s'applique pas très bien à l'Amazonie» (Ibid. : 512).

17. "Il exprime néanmoins le désir de redéfinir les notions pour un débat anthropologique plus large, et c'est précisément là que les difficultés commencent [...]. Plutôt que d'envisager la possibilité que sa définition du cannibalisme ne s'applique pas au cas des Rock Crees, Fausto semble accuser Brightman de mal comprendre son propre matériel ethnographique " (Vaté, in Fausto $2007: 520$ ).

18. "Mon objectif principal n'est pas ici de montrer qu'un modèle du don nord-américain est relativement moins productif en Amazonie » (Fausto 2007 : 500). 
bien fondée empiriquement ou plus confuse dans ses objectifs que la comparaison intrarégionale de Fausto, mais plutôt qu'elle produit un effet subtilement différent.

En opposant une description minutieusement fondée de l'Amazonie à des analyses nord-américaines dont le fondement empirique est quant à lui mis entre parenthèses, ce type de comparaison latérale évite d'avoir à s'interroger sur la validité des travaux de collègues travaillant dans d'autres contextes. Qu'ils soient littéraux ou littéraires, leur intérêt est le même: obtenir par contraste une logique différente.

Ce type de comparaison latérale fait plutôt penser à la réinvention postpluraliste de la comparaison frontale: comme nous l'avons vu précédemment, l'élément clé de cette récupération du frontal était le fait que, littéraire ou littéral, l'Occident était à nouveau autorisé à jouer le rôle d'homme de paille pouvant être utilisé pour élaborer de nouveaux concepts et ouvrir de nouvelles perspectives (Strathern 1981). Il y a néanmoins une différence importante. Dans le cas frontal, la question de savoir si l'image de l'Occident est littérale ou littéraire n'a pas à être tranchée, car même le littéraire relève, en dernière instance, du littéral. Soit parce que (selon une lecture politique) les mythes de l'Occident ont eux-mêmes un impact important qui doit être contré, soit parce que (selon une lecture épistémologique), la propre activité littéraire de l'anthropologue est, au fond, la manifestation littérale de l'Occident au travail.

En revanche, quand il refuse de juger si les lectures de l'animisme basées sur les matériaux nord-américains sont littéralement vraies ou ne sont que les effets littéraires du point de vue de leurs auteurs, Fausto n'est pas obligé de prendre position. En d'autres termes, la marque distinctive de ce type de comparaison latérale est qu'elle ne peut elle-même résoudre la question de la portée de ses conclusions. Si les autres études étaient correctes, alors les résultats de l'analyse permettraient d'identifier une spécificité régionale - les choses sont différentes en Amazonie et il existe donc des différences cruciales au sein de la catégorie générale "animisme». Si les redéfinitions proposées du cannibalisme et du dualisme corps/esprit parvenaient à "s'appliquer » à l'Amérique du Nord également, alors leur portée serait celle d'une généralisation théorique: une redéfinition des systèmes animistes. Mais, fondamentalement, l'heuristique elle-même ne permet pas de trancher entre les deux.

Autrement dit, Fausto rend explicite une démarche que de nombreux anthropologues font implicitement - quand, en dehors de notre zone géographique d'expertise, nous avons tendance à faire abstraction de la validité des travaux dont nous nous inspirons pour un éclairage comparatif. Rendre la démarche explicite, en revanche, c'est pointer ses limites 
intrinsèques. Encore une fois, ces limites ne posent problème que si nous oublions le fait que les études anthropologiques sont interdépendantes. Ce type d'heuristique latérale, par définition irrésolue, n'est qu'une des démarches mobilisées dans le processus continuel de redéfinitions régionales et conceptuelles. La question de savoir si les analyses existantes du matériau nord-américain sont correctes ou convaincantes ne peut trouver de réponse en se référant au matériau amazonien. Il reviendra aux nord-américanistes, à leur tour, de décider si cela constitue ou non une critique de leurs positions.

Une troisième forme, encore différente, de comparaison latérale traverse l'argumentation de Fausto. C'est l'usage de modèles théoriques inspirés des travaux de Marilyn Strathern en Mélanésie: modèles des personnes "dividuelles », de l'agencéité relationnelle et de la transaction de perspectives ${ }^{19}$. Ces modèles sont censés pouvoir «s'appliquer" à l'Amazonie, précisément là où les modèles nord-américains sont censés ne pas le pouvoir. Dans ce cas, cependant, l'appartenance régionale ne fait jamais l'objet d'une discussion : le mot "Mélanésie " ne figure même pas dans cet article. Il serait difficile, et sinon malhonnête, de lire cet article comme s'il prétendait que les Amazoniens ressemblent plus à des Mélanésiens qu'à des Indiens d'Amérique du Nord. En fait, cette dernière forme de comparaison latérale met totalement entre parenthèses ces préoccupations inter-régionales, tout comme le second genre de comparaison latérale mettait entre parenthèses la validité empirique des travaux nord-américains. Mais elle ne le fait pas de la même façon.

Nous avons ici une autre heuristique classique de comparaison latérale, aussi ancienne que la «comparaison contrôlée » d'Eggan : la mise en évidence de similitudes entre entités dont la distance géographique et culturelle est extrême permet précisément à l'auteur de mettre de côté les questions d'origine commune ou de parenté. Pour les auteurs fonctionnalistes classiques, ces analogies entre entités distantes incarnaient l'habileté comparatiste de l'anthropologie à son apogée généralisatrice (Radcliffe-Brown 1951 ; Nadel 1951) : libérés de la question des origines communes, ils ne parlaient que des lois universelles du fonctionnement social. Dans l'article de Fausto, en revanche, ces analogies visent à obtenir un résultat tout à fait différent. En faisant abstraction de son appartenance régionale, un concept élaboré à partir d'une enquête locale (par exemple,

19. Par exemple: "J'ai préféré considérer la personne comme un amalgame d'activité et de passivité, comme "quelqu'un" qui contient deux perspectives possibles dans une relation de prédation. Le passage de la puissance à l'acte, de la tension prédatrice à l'acte prédateur, est ce qui produit la disjonction de ces perspectives en parties détachables, parties qui peuvent alors être traitées» (Fausto $2007: 513$ ). 
les personnes «dividuelles » de Strathern) peut «voyager » et être utilisé dans un contexte tout à fait différent tout en échappant à la généralisation ${ }^{20}$. Ce type de comparaison latérale vient cependant parasiter la comparaison s'intéressant aux spécificités régionales. Elle transfere un concept d'un lieu à un autre, mais ne permet pas de caractériser la région d'application.

Cet article de Fausto est sans doute un exemple extrême de par l'étendue et la variété des comparaisons latérales qu'il déploie, et de par l'attention qu'il consacre à la construction d'une aire culturelle. Mais il illustre bien tout ce qui se cache derrière la référence, souvent trop rapide, aux travaux d'autres anthropologues ayant enquêté sur le même "sujet» en dehors de la "région" étudiée. Il montre, en gros plan pour ainsi dire, la présence constante de la comparaison latérale dans l'anthropologie contemporaine, la façon dont elle favorise le dialogue entre nous tout en tissant des liens, mais aussi les nombreuses formes qu'elle revêt et les décalages épistémiques que cette diversité implique.

Je tiens, encore une fois, à insister sur le fait que cette analyse des trois formes de comparaison latérale en tant qu'heuristiques distinctes est, bien sûr, la mienne. Fausto lui-même ne s'intéresse pas à la manière dont ces diverses démarches doivent être reliées, si elles répondent aux mêmes paramètres épistémologiques, ni même les enjeux liés à leur combinaison. D’une manière générale, elles sont toutes considérées comme une méthode anthropologique parmi d'autres, à savoir la comparaison. Cela dit, j'espère que ces lignes font clairement ressortir que l'on a affaire à un ensemble d'heuristiques très différentes. Une discussion plus approfondie sur ce qui les caractérise, leurs points forts et leurs limites, à la fois pour chacune d'entre elles et dans leurs diverses combinaisons, serait un projet digne d'être lancé si nous souhaitons aboutir à des analyses solides, convaincantes et novatrices.

$$
\mathrm{V}
$$

«Our truth is the intersection of independent lies» (Levins, cité in Wimsatt $2007:$ 43).

En ramenant le latéral au premier plan, on fait évoluer, aussi, notre perception de la comparaison frontale. J'ai mentionné plus haut que l'on pouvait caractériser provisoirement la comparaison frontale comme

20. Comme l'écrit Marilyn Strathern dans un texte qui répond au travail de Fausto par une caractérisation des différences amazonienne-mélanésienne : «la capacité collective de l'anthropologie à embrasser la perspective la plus vaste ne peut s'exercer qu'à des fins de particularisation des exposés spécifiques" (2012: 13). 
l'opération qui rend l'étrange familier et le familier étrange, tandis que la comparaison latérale permet d' "approfondir " notre investigation. Dans cette conclusion, je voudrais donner à ces caractérisations provisoires un contour un peu plus précis.

À première vue, ces deux heuristiques travaillent à partir de et avec des entités que l'on suppose être assez distinctes pour être comparées. Mais, en même temps, toutes deux contribuent également à démontrer le fait même de ce caractère distinctif. C'est toujours par la comparaison que les anthropologues ont établi qu'il y a des domaines distincts à comparer. Toutefois, la comparaison latérale et la comparaison frontale procèdent de différentes façons et avec des effets différents.

Dans la comparaison frontale, on obtient une différence entre deux domaines, l'un d'entre eux incluant la perspective qui opère la différenciation. D'où cette dualité caractéristique de la formulation d'EvansPritchard: l'hinterland correspond aux «concepts et [au] système de valeurs qui sont ceux de [la] culture [de l'anthropologue] et des connaissances particulières à la discipline qu'il pratique" (1969 [1951] : 80). En d'autres termes, l'hinterland est à la fois un domaine de comparaison, tout comme l'est l'ethnographie (« nous avons notre culture comme ils ont la leur»), et un ensemble de connaissances anthropologiques confrontées à une nouvelle réalité empirique. Tout en gardant la forme d'une grille de lecture (perfectible) grâce à laquelle l'ethnographie peut être comprise, cette heuristique ouvre la possibilité plus radicale de voir la comparaison frontale remettre en question les termes mêmes avec lesquels on fait la comparaison. La grille de lecture, par définition, est déjà relativisée. Telle est la clé de la dimension intrinsèquement polémique de la comparaison frontale. Autrement dit, l'apport distinctif de la comparaison frontale réside dans sa capacité à déstabiliser les catégories d'analyse servant à aborder l'« objet ».

Le point de défaillance systématique de la comparaison frontale, par ailleurs, est qu'elle a peu à dire sur la forme et le contour des entités ainsi mises en contraste. Elle élude ce problème en partant d'un contraste fondé sur la propre expérience de l'anthropologue. De quoi ce contraste est-il représentatif et quelle entité vient-il signifier? La comparaison frontale nous permet de laisser cela dans le vague. Autrement dit, cette opération nous oblige à postuler une seule entité de chaque côté, en faisant abstraction de leur multiplicité interne.

La comparaison frontale en anthropologie correspond à la description, par Wimsatt, des effets étonnamment informatifs d'observations incertaines et contradictoires, tant que l'on postule un (et un seul) "objet" et un (et un seul) «appareil expérimental»: 
«Étant donné les observations imparfaites de quelque-chose-que-nous-ne-connaissonspas, en utilisant un appareil expérimental chargé de préjugés-que-nous-risquons-dene-pas-comprendre, nous pouvons obtenir une meilleure compréhension à la fois de l'objet (qui doit être, après tout, cette chose même dont les propriétés peuvent produire ces résultats divergents dans les détecteurs) et de l'appareil expérimental (qui est formé, après tout, de ces éléments qui peuvent être affectés de façon divergente par une même chose)» (2007: 58).

Dès lors, la sur-généralisation de l'«Autre » et la tendance à prendre toujours les mêmes boucs émissaires comme caractéristiques du "Soi » ne sont rien d'autre que le risque inhérent à ce procédé anthropologique, sa «marque " distinctive. C'est pourquoi la critique qui objectera qu'il y a plus de complexité au sein de l'hinterland, ou que la description ethnographique est trop générale, ou encore que, à bien des égards, "eux " et "nous" se ressemblent beaucoup, sera perçue comme inintéressante, tatillonne, passant à côté de l'esprit de la pratique ${ }^{21}$. En fait, je dirais que les anthropologues ont pu percevoir à nouveau l'intérêt de la comparaison frontale dans une atmosphère conceptuelle postpluraliste, précisément parce qu'elle n'a jamais accordé beaucoup d'importance aux questions de délimitation ${ }^{22}$.

En revanche, dans la comparaison latérale, ce qui est en jeu est justement la circulation de concepts à travers un paysage constitué d'entités (sociétés, institutions, événements, etc.) et, en même temps, la division et le regroupement, la configuration et la reconfiguration de ces entités. À première vue, la procédure latérale semblerait moins révolutionnaire. Là encore la grille de lecture est constamment mise en question et en danger, seulement, cette fois, elle l'est collectivement. Chaque cas nouveau ajoute aux difficultés de l'ensemble, ou oriente les analyses dans une autre direction. Au moment où les domaines de comparaison (aires régionales ou thématiques) semblent se tenir sagement côte à côte, la comparaison latérale révèle plus de différences internes ou de connexions inattendues entre elles. Au moment où la connaissance semble s'être stabilisée, la comparaison latérale génère de nouvelles questions, de nouveaux problèmes.

21. "Les "Balkans" ressemblent plutôt à la "Mélanésie" ou à l' "Amazonie" dans la mesure où il s'agit d'un champ épistémique susceptible d'innombrables analyses. (À d'autres égards, bien sûr, je plaide pour le contraste.) Il ne sert à rien d'objecter que ce sont des généralisations sauvages ou de soulever des points de contradiction, puisque les deux démarches sont englobées dans le terme générique " (Strathern $2011: 98$ ).

22. En dernière instance, pour forcer légèrement le trait, on pourrait dire que le bénéfice fondamental de la comparaison frontale - rendre l'étrange familier et le familier étrange - n'est pas nécessairement lié au domaine de l'ethnographie classique. Une tentative réussie de comparaison frontale perturbant nos vérités préconçues est celle d'Anna Tsing, qui explicite sous forme littéraire le point de vue d'une spore de champignon (Tsing 2014). Les fameux « Nacirema (Miner 1956) en sont une autre. 
La limite de la comparaison latérale, son point de défaillance, est son incapacité inhérente à se passer, d'une façon ou d'une autre, d'un cadre pluraliste. C'est en soi une limite sérieuse, qui a contribué à rendre si difficile la revitalisation de la comparaison latérale après la crise épistémique des années 1980. Et pourtant, associé à la comparaison frontale, ce questionnement pluraliste incessant s'avère un perturbateur fructueux. Il rappelle avec force que "dans chaque peuple il y a d'autres peuples " (Candea 2011 : 149) et s'oppose à la tendance de la comparaison frontale à se fixer sur la démonstration, toujours récurrente, de l'«autre» en tant qu’image inversée du «nous» (Trouillot 1991).

Dernier complément important à cette conclusion: la division et le regroupement opérés par la comparaison latérale ont lieu à partir du point de vue d'un tiers, mais qui reste fondamentalement collectif: la perspective partagée par les anthropologues spécialistes d'une région (comme l'Amazonie) ou d'un thème (comme l'animisme) particuliers, et qui échangent sur leurs domaines de compétence ou au-delà. Et, bien sûr, cette manière de procéder fait, défait et refait simultanément leurs spécialisations. Les comparaisons latérales sont nécessairement limitées par le fait de n'avoir qu'une stabilité temporaire - elles invitent à d'autres questionnements latéraux. Pour reprendre encore une fois les propos de Wimsatt:

"Les clauses du type ceteris paribus ont leur charme. Elles nous donnent accès aux régularités et aux modifications que nous savons à notre disposition tout en nous rappelant les exceptions - les fluctuations ou les déviations d'ordre macroscopique qui ouvrent la voie à une compréhension plus profonde. Cette forme cognitive (schéma général + exceptions) et ses semblables (similitudes larges + différences connexes, modèles + qualifications, etc.) sont profondément ancrées dans la structure de notre organisation de connaissances fondée sur des cas: "Ceci ressemble à cela (que vous connaissez déjà) mais avec les différences suivantes". Elles sont porteuses de la micro-structure de l'apprentissage cumulatif» (2007: 33).

En revanche, en dernière instance, la comparaison frontale est en soi une procédure expérimentale individuelle, une "équation personnelle » (Kuklick 2011) : elle rend compte de la transformation engendrée par l'expérience de l'altérité chez un anthropologue et sur sa propre perception du familier. En faisant appel à un hinterland, la comparaison frontale implique les lecteurs dans un «nous » perspectif (Pina Cabral 2006 : 667), dont la forme demeure indécise et discutable: l'hinterland est toujours à la fois un "nous " partagé et une position que l'anthropologue lui-même laisse derrière lui au moment même où il le formule.

Les deux heuristiques ne peuvent se passer l'une de l'autre. Leurs faiblesses autant que leurs forces se complètent et se soutiennent mutuellement. La comparaison frontale ne peut "s'étendre" que grâce aux 
comparaisons latérales. Celles-ci lui fournissent son matériau, lui permettent de voyager et, finalement, jugent de sa pertinence. La comparaison latérale commence et finit avec des remises en questions frontales de ses procédés de cadrage toujours réinventés. Dans la comparaison frontale, nous nous mettons nous-mêmes à l'épreuve. Dans la comparaison latérale, nous nous mettons mutuellement à l'épreuve. Leur danse est la danse de la connaissance anthropologique. Mais, alors que, d'un côté, nous sommes désormais bien armés pour penser la force, les promesses et les limites de la comparaison frontale, de l'autre, la réflexion épistémologique pour "réagencer" la comparaison latérale ne fait que commencer (Strathern 2012 ; Descola 2005; Pedersen \& Nielsen 2013).

\section{Traduit de l'anglais par Franck Lemonde** \\ University of Cambridge \\ Division of Social Anthropology, Cambridge (Angleterre) mc288@cam.ac.uk}

MOTS CLÉS/KEYWORDS : épistémologie/epistemology - méthode comparative/comparative method - comparaison frontale/frontal comparison - comparaison latérale/lateral comparison heuristique/heuristic - cannibalisme/cannibalism.

** $\mathrm{Y}$ compris pour les citations extraites d'ouvrages en anglais $[N d l r]$. 


\section{RÉFÉRENCES CITÉES}

Anderson, Amanda

2001 The Powers of Distance.

Cosmopolitanism and the Cultivation

of Detachment. Princeton, Princeton

University Press.

Asad, Talal

1986 «The Concept of Cultural

Translation in British Social

Anthropology ", in James Clifford \&

George E. Marcus, eds, Writing Culture. The

Poetics and Politics of Ethnography. Berkeley,

University of California Press : 141-164.

Beattie, John

1972 [1964] Introduction à l'anthropologie

sociale [trad. de: Other Cultures.

Aims Methods and Achievements

in Social Anthropology]. Trad. de l'anglais

par Georgette Rintzler-Neuburger. Paris,

Payot («Bibliothèque scientifique» 54).

\section{Candea, Matei}

2011 «Endo/Exo», Common Knowledge

$17: 146-150$.

\section{Carrier, James G.}

1992 "Occidentalism : The World Turned Upside-Down ", American Ethnologist 19 (2) : 195-212.

\section{Chakrabarty, Dipesh}

2009 [2000] Provincialiser l'Europe.

La pensée postcoloniale et la différence

historique. Trad. par Olivier Ruchet

et Nicolas Vieillescazes. Paris, Amsterdam.

Descola, Philippe

2005 Par-delà nature et culture.

Paris, Gallimard ("Bibliothèque

des sciences humaines »).

Evans-Pritchard, Edward E.

1950 «Social Anthropology. Past

and Present: The Marett Lecture, 1950 ", Man 50: 118-124.
1969 [1951] Anthropologie sociale. Trad.

de l'anglais par Monique Manin.

Postface de Michel Panoff. Paris, Payot

(«Petite bibliothèque Payot»132).

Fabian, Johannes

2006 [1983] Le Temps et les autres.

Comment l'anthropologie construit son objet.

Trad. de l'anglais par Estelle Henry-

Bossonney et Bernard Müller. Avant-propos

d'Alban Bensa. Postface de Johannes Fabian.

Toulouse, Anacharsis ("Essais»).

Fausto, Carlos

2007 «Feasting on People : Eating Animals and Humans in Amazonia ",

Current Anthropology 48 (4) : 497-530.

Fortes, Meyer \& Edward E. Evans-Pritchard, eds 1964 [1940] Systèmes politiques africains.

Trad. de l'anglais par Paul Ottino. Avantpropos d'Hubert Deschamps. Préf. de A. R. Radcliffe-Brown. Paris, Presses universitaires de France («Études ethnographiques»).

\section{Gad, Christopher}

2012 «What We Talk About When We Talk About Sailor Culture : Understanding Danish Fisheries Inspection Through a Cult Movie ", Culture Unbound: Journal of Current Cultural Research 4 : 367-392.

Gad, Christopher \& Casper Bruun Jensen

2016 "Lateral Comparisons ", in Joe Deville, Michael Guggenheim \& Zuzana Hrdličková, eds, Practising Comparison. Logics, Relations, Collaborations. Manchester, Mattering Press [à paraître].

\section{Geertz, Clifford}

1996 [1988] Ici et là-bas. L'anthropologue comme auteur. Trad. de l'amér. par Daniel Lemoine. Paris, Métailié (« Leçons de choses»).

Handler, Richard

2009 «The Uses of Incommensurability in Anthropology ", New Literary History 40 (3) : 627-647. 


\section{Hartog, François}

1980 Le Miroir d'Hérodote. Essai sur

la représentation de l'autre. Paris, Gallimard

( Bibliothèque des histoires ").

Henare, Amiria, Martin Holbraad

\& Sari Wastell, eds

2007 Thinking Through Things. Theorising

Artefacts Ethnographically.

London, Routledge.

Holbraad, Martin

2012 Truth in Motion. The Recursive Anthropology of Cuban Divination. ChicagoLondon, University of Chicago Press.

Holbraad, Martin \& Morten Axel Pedersen

2009 «Planet $\mathrm{M}$ : The Intense Abstraction of Marilyn Strathern ", Anthropological

Theory 9 (4) : 371-394.

Holbraad, Martin \& Morten Axel Pedersen

\& Eduardo Viveiros de Castro

2014 «The Politics of Ontology:

Anthropological Positions ", Cultural

Anthropology Online [http://culanth.org/

fieldsights/462-the-politics-of-ontology-

anthropological-positions].

Holý, Ladislav

1987 « Description, Generalization and Comparison : Two Paradigms ", in Ladislav Holý, ed., Comparative Anthropology... : $1-21$.

Holý, Ladislav, ed.

1987 Comparative Anthropology.

Oxford-New York, Blackwell.

Ingold, Tim

1993 "The Art of Translation in a

Continuous World », in Gísli Pálsson, ed.,

Beyond Boundaries. Understanding,

Translation and Anthropological Discourse.

Oxford-Providence, Berg : 210-230.

2000 The Perception of the Environment.

Essays on Livelihood, Dwelling \& Skill.

London-New York, Routledge.
Iteanu, André \& Ismaël Moya

2015 "Mister D. : Radical Comparison, Values, and Ethnographic Theory ", Hau. Journal of Ethnographic Theory 5 (1) : 113-136.

Kuklick, Henrika

2011 «Personal Equations: Reflections on the History of Fieldwork, with Special Reference to Sociocultural Anthropology ", Isis 102 (1) : 1-33.

\section{Lazar, Sian}

2012 «Disjunctive Comparison :

Citizenship and Trade Unionism in Bolivia and Argentina ", Journal of the Royal

Anthropological Institute 18 (2) : 349-368.

\section{Lévi-Strauss, Claude}

1962 La Pensée sauvage. Paris, Plon.

\section{Lewis, Oscar}

1955 "Comparisons in Cultural Anthropology ", in William L. Thomas Jr, ed., Yearbook of Anthropology. Chicago, University of Chicago Press : 259-292.

Lienhardt, Godfrey

1953 "Modes of Thought in Primitive Society ", New Blackfriars 34 : 269-277.

Malinowski, Bronislaw

1925 "Magic, Science and Religion", in Joseph Needham, ed., Science, Religion and Reality. New York, Macmillan Company : 19-84.

Marcus, George E. \& Michael M. J. Fischer 1999 [1986] Anthropology as Cultural Critique. An Experimental Moment in the Human Sciences.

Chicago, Chicago University Press.

Miner, Horace

1956 "Body Ritual Among the Nacirema", American Anthropologist 58 (3) : 503-507.

Mol, Annemarie

2002 The Body Multiple. Ontology in Medical Practice. Durham, Duke University Press. 
Nadel, Siegfried F.

1951 The Foundations of Social

Anthropology. London, Cohen \& West.

Navaro-Yashin, Yael

2009 «Affective Spaces, Melancholic

Objects : Ruination and the Production

of Anthropological Knowledge ", Journal of

the Royal Anthropological Institute 15 (1) : 1-18.

\section{Pedersen, Morten Axel \& Morten Nielsen}

2013 «Trans-Temporal Hinges: Reflections on an Ethnographic Study of Chinese

Infrastructural Projects in Mozambique and

Mongolia ", Social Analysis 57 (1) : 122-142.

Pina-Cabral, João de

1992 "Against Translation: The Role of the Researcher in the Production of Ethnographic Knowledge ", in João de Pina-Cabral \& John Campbell, eds, Europe Observed. London, Macmillan : 1-23.

2006 "Anthropology Challenged:

Notes for a Debate", Journal of the Royal Anthropological Institute 12 (3) : 663-673.

2009 "The All-or-Nothing Syndrome and the Human Condition ", Social Analysis 53 (2) : 163-176.

Pitt-Rivers, Julian A.

1954 The People of the Sierra. Introd.

by E. E. Evans-Pritchard. London,

Weidenfeld \& Nicholson.

\section{Radcliffe-Brown, Alfred R.}

1951 "The Comparative Method in Social Anthropology ", Journal of the Anthropological Institute of Great Britain and Ireland 81 (1-2) : 15-22.

Radcliffe-Brown, Alfred R. \& Daryll Forde, eds 1953 [1950] Systèmes familiaux et matrimoniaux en Afrique. Trad. par Marcel Griaule. Paris, Presses universitaires de France ("Bibliothèque de sociologie contemporaine ").

\section{Said, Edward W.}

2003 [1978] Orientalism. London, Routledge \& Kegan Paul.

\section{Salmon, Gildas}

2013 Les Structures de l'esprit. Lévi-Strauss et les mythes. Paris, Presses universitaires

\section{Strathern, Marilyn}

1981 "Culture in a Netbag: The Manufacture of a Subdiscipline in Anthropology ",

Man. 16 (4) : 665-688.

1987 "The Limits of Auto-Anthropology ", in Anthony Jackson, ed., Anthropology at Home. London, Tavistock : 59-67.

1988 The Gender of the Gift. Problems with Women and Problems with Society in Melanesia. Berkeley, University of California Press.

2004 [1991] Partial Connections.

Savage, Rowman \& Littlefield.

2011 «Binary License ", Common

Knowledge 17 (1) : 87-103.

2012 "Eating (and Feeding)", Cambridge

Journal of Anthropology 30 (2) : 1-14.

\section{Trouillot, Michel-Rolph}

1991 «Anthropology and the Savage Slot:

The Poetics and Politics of Otherness ",

in Richard C. Fox, ed., Recapturing Anthropology. Working in the Present.

Santa Fe, School of American Research Press.

\section{Tsing, Anna Lowenhaupt}

2014 «Strathern Beyond the Human: Testimony of a Spore ", Theory, Culture \& Society 31 (2-3) : 221-241.

\section{Van Fraassen, Bas C.}

1980 The Scientific Image. Oxford, Clarendon Press / New York, Oxford University Press.

\section{Viveiros de Castro, Eduardo}

1998 "Cosmological Deixis and Amerindian Perspectivism ", Journal of the Royal Anthropological Institute 4 (3) : 469-488.

2004a «Exchanging Perspectives:

The Transformation of Objects into

Subjects in Amerindian Ontologies ", Common Knowledge 10 (3) : 463-484. 
2004b « Perspectival Anthropology

and the Method of Controlled

Equivocation", Tipití 2 (1) : 3-22.

2011 «Zeno and the Art of Anthropology:

Of Lies, Beliefs, Paradoxes, and Other

Truths", Common Knowledge 17 (1) :

128-145.

Wagner, Roy

1981 [1975] The Invention of Culture.

Chicago, University of Chicago Press.
Willerslev, Rane

2004 «Not Animal, Not Not-Animal :

Hunting, Imitation and Empathetic

Knowledge Among the Siberian Yukaghirs ", Journal of the Royal Anthropological Institute $10(3)$ : 629-652.

Wimsatt, William C.

2007 Re-Engineering Philosophy for Limited

Beings. Piecewise Approximations to Reality.

Cambridge, Harvard University Press.

RÉSUMÉ/ABSTRACT

Matei Candea, De deux modalités de comparaison en anthropologie sociale. - Cet article se propose de reconsidérer l'histoire et l'état actuel de la comparaison en anthropologie, en établissant une distinction entre deux heuristiques comparatives : la " comparaison frontale " d'une part, où une entité ethnographique étrangère est opposée à un univers supposé comme familier, et, d'autre part, les "comparaisons latérales", où un certain nombre de cas sont confrontés les uns aux autres. Alors que la comparaison frontale a monopolisé l'attention et les questionnements épistémiques pendant les dernières décennies, les discussions méthodologiques et épistémologiques concernant la comparaison latérale ont été laissées de côté, étant jugées comme relevant d'une approche positiviste stérile et datée. Par contraste, cet article examine la persistance, la vitalité et la diversité de la comparaison latérale; il souligne l'interdépendance entre les deux approches heuristiques tout en affirmant qu'il est temps de prendre au sérieux la comparaison latérale.
Matei Candea, Going Full Frontal, or the Elision of Lateral Comparison in Anthropology. - This article proposes a reconsideration of the history and contemporary state of comparison in anthropology, by introducing a distinction between two comparative heuristics: on the one hand, "frontal comparison", in which an unfamiliar ethnographic entity is contrasted to a putatively familiar background, and on the other, "lateral comparisons", in which a number of "cases " are laid side by side. While frontal comparison has monopolised anthropologists' epistemic attention and concern over recent decades, methodological and epistemological discussions of lateral comparison have mostly been relegated to the doldrums of an outdated positivism. By contrast, this article examines the persistence, vitality and diversity of lateral comparison, foregrounds the interdependence of the two heuristics, and argues that it is time to bring lateral comparison back into the light. 